\title{
Research and Application of WCF Technology in Data Acquisition of Ultra-high Speed Packaging Machine
}

\author{
Jie Qian ${ }^{1, a}$, Li Ming Zhu ${ }^{1}$, Zhen Xun Jin ${ }^{1}$ and Huan Le ${ }^{1}$ \\ ${ }^{1}$ China Tobacco Zhejiang Industrial Co. LTD., Hangzhou, China
}

\begin{abstract}
By introducing WCF technology on data acquisition of ultra-high speed packaging machine, data acquisition system reads dates of machine in polling mode through the WCF client, which can achieve accurate data collection, and effectively isolate the data acquisition system and the machine control system. It enhances the security of data interaction between systems, but also reduces the coupling degree between systems.
\end{abstract}

Keywords: WCF, H1000, Data Acquisition, Ultra-high Speed Packaging Machine.

\section{Introduction}

In the Eleventh Five ex situ technological transformation, Hangzhou Cigarette Factory introduces ultra-high speed packaging machine, which is designed and manufactured in Italian GD Company. This is one of the most technologically advanced ultra-high speed cigarette packaging machine, with the production capacity of 1,000 packages per minute, which is one of the key models that the tobacco companies will introduce [1]. The data acquisition system of Hangzhou cigarette factory will acquire production data of packaging machine, which will used to analysis operating status, and also analysis input-output and raw materials consumption. The following paper will divided into four parts: technology analysis, communication interface protocol analysis, development and design, summarize.

\section{Technology analysis}

WCF is the short of Windows Communication Foundation, which is a communication technology framework to create and run a distributed system, it provides a service-oriented system to build secure, reliable, cross-platform distribution solutions [2]. The communication model structure of WCF is shown in Fig. 1. Address, binding and contract constitutes an endpoint. The endpoint client and the server is the basis for message exchange. The server public endpoint. When the endpoint match between client and server, both will be able to interact through messages [3]. WCF technology has been widely used in the field of distributed systems, business intelligence and data synchronization [4-6].

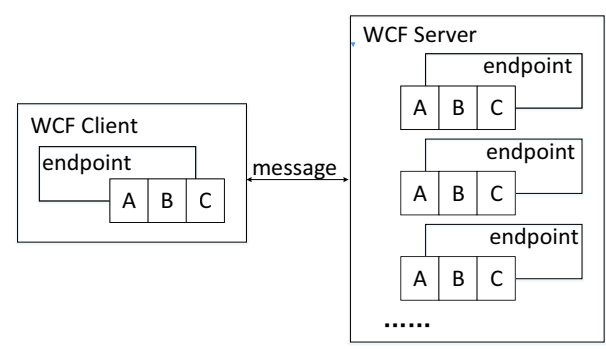

Fig. 1: communication model structure of

WCF

GD Company uses new technology in their ultra-high speed packaging machine. They don't use MICROII system which is their own development electric control system, but use the

\footnotetext{
${ }^{\mathrm{a}}$ Corresponding author: qianj@zjtobacco.com
} 
production of Beckhoff which is much more popular recently. Corresponding HMI program is also replace by aHMI programs that is developed with .NET. HMI program communicates with data acquisition system through WCF.

\section{Communication protocol analysis}

interface

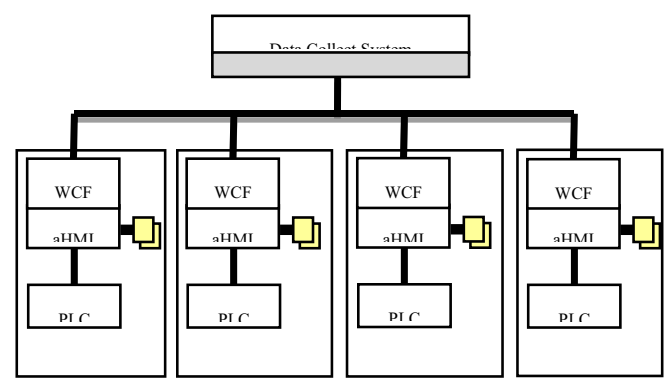

Fig. 2: the communication architecture

As shown in Fig. 2, the communication protocol is based on client-server architecture, while the aHMI program as WCF server, and data acquisition system as WCF client. We will use WSDualHttpBinding which is a secure and interoperable binding, and suits for communication in duplex service agreements or SOAP. Due to the use WSDualHttpBinding, we need to open two HTTP channels, one for service and one for the callback. Therefore, you need to configure two HTTP address, and the HTTP port number is configured as 8005 in GD.

\section{Development and design}

Ultra-high speed packaging machine consists of four parts which are H1000, W1000, C800BV and Material Automation, data acquisition systems via WCF client communicate with WCF services of four parts respectively. Interactive data between systems can be divided into three categories: machine information, statistical data and production grades. The details is shown in Table 1.

\begin{tabular}{|l|l|}
\hline category & details \\
\hline $\begin{array}{l}\text { machine } \\
\text { information }\end{array}$ & machine status, machine speed, machine user \\
\hline statistical data & $\begin{array}{l}\text { Machine rated speed, qualified products, } \\
\text { excluding products, machine time (production, } \\
\text { low speed, wait, internal stop and external stop, } \\
\text { etc.), set production classes, material } \\
\text { consumption, the date and time settings, machine } \\
\text { failure statistics, history of mechanical failure }\end{array}$ \\
\hline $\begin{array}{l}\text { production } \\
\text { grades }\end{array}$ & The setting of production grade \\
\hline
\end{tabular}

Table 1: details of the interactive data

After acquiring data from four aHMI server of each machine, Data acquisition system needs write data to system database in accordance with a standardized. System reads the data of machine in polling mode through WCF client. The details is shown in Fig. 4.

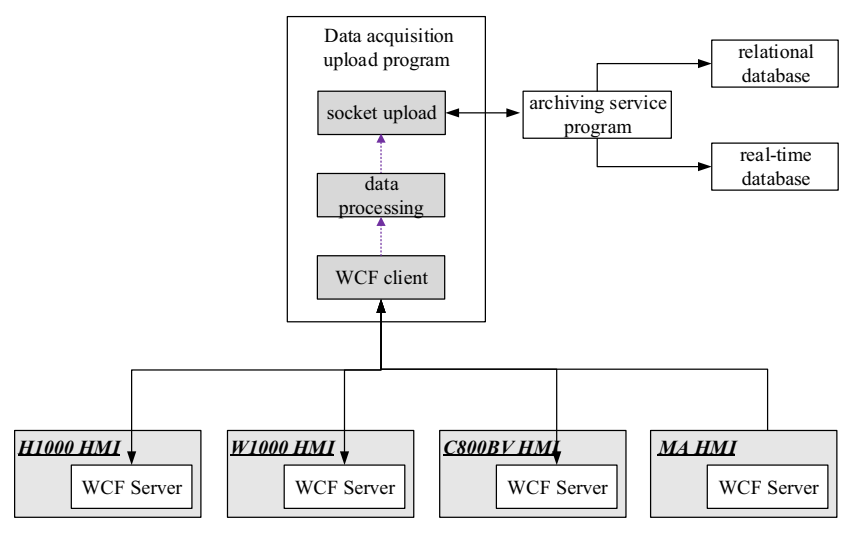

Figure 3: the details of data acquisition

\section{Summarize}

Through the application of WCF technology in ultra-high speed packaging machine, the system can successfully acquire machine information, statistics and production grades, etc. The control system only open binding address and service, the external system cannot interfere with internal control procedures of machine, this messaging model is also effective to reduce the 
degree of correlation between the two data exchange systems, to enhance the security between systems when data interaction, but also reduces the associated coupling between systems.

\section{REFERENCES}

[1] STATE TOBACCO MONOPOLY ADMINISTRATION, The policy of "12th Five-Year" technology and equipment in the tobacco industry[R].

[2] Jiang Jinnan, WCF Technical Analysis .Beijing: publishing house of electronics industry, 2009.

[3] Yan Shang, Huang Zhangchan. WCF: New Fundament of Communication Technology Based on Windows Platform [J]. Computer \& Digital Engineering, 2008(4):86-89.

[4] Wu Qingshou. Research and Implementation on the Distributed System Model of WCF [J]. Jilin Normal University Journal(Natural Science Edition), 2012(3):61-64.

[5] Lin Xin, Zhou Yonggang, Yan Hua. Research and Application of Business Intelligence System Based on WCF [J]. Computer Technology and Development, 2010(7): 223-226.

[6] Zhang Qingfeng, Zhang Fengqin, Wang Lei. Design and Realization of Data Synchronization Model Based on WCF [J]. Journal of Shanxi Datong University(Natural Science Edition), 2013, 29(2): 9-11. 